\title{
CLINICAL AND ANGIOGRAPHIC PROFILE OF FEMALES IN CENTRAL INDIA PRESENTING WITH ACUTE CORONARY SYNDROME
}

\author{
Tribhuwan Nath Dubey1, Abhinav Kumar ${ }^{2}$ \\ 1 Professor and HOD, Department of Medicine, Gandhi Medical College, Bhopal. \\ ${ }^{2} 3^{\text {rd }}$ Year Postgraduate Trainee, Department of Medicine, Gandhi Medical College, Bhopal.
}

\section{ABSTRACT}

Coronary Artery Disease (CAD) is the leading cause of death in women. There is a rapid increase in rates of CAD in Indian subcontinent, so there is a need to clarify the epidemiology and profile of CAD especially in females.

\section{OBJECTIVE}

To study the presentation and prevalence of risk factors in females presenting with ACS and to correlate their impact on Coronary Angiography (CAG) profile.

\section{METHODS}

The study was an observational study carried out at authors institute for 1 year (December 2014 to November 2015). The study group comprised of 92 female patients presenting with first episode of ACS (evidence of unstable angina/myocardial infarction). The following data was analysed in all subjects: Age, Menopausal status, Symptoms, Killip class, Smoking/tobacco chewing, Family history (FH) of CAD, Hypertension, Diabetes mellitus, Waist-hip ratio, Dyslipidaemia, Thyroid profile, ECG, CPK-MB, and CAG.

\section{RESULTS}

We found that the mean age of females in our study was $56.7 \pm 10.43$ years with STEMI in $63.1 \%$, UA in $30.4 \%$, and NSTEMI in 5.5\%. 81.5\% were postmenopausal, $62 \%$ were hypertensive, and 34.8\% were diabetic. Dyslipidaemia (90.2\%) followed by obesity $(68.5 \%)$ were most prevalent risk factors. $13 \%$ had overt hypothyroidism and $23.9 \%$ had subclinical hypothyroidism. $31.5 \%$ had a positive FH of CAD. Most common coronary artery involved was LAD (58.5\%) followed by RCA (40\%), and LCx (34.2\%). LMCA disease was seen in $2.8 \%$ of females. Of the risk factors, dyslipidaemia, hypertension, and age $\geq 55$ years correlated significantly ( $\mathrm{p}$ value $<0.05$ ) with presence of obstructive CAD on CAG.

\section{CONCLUSION}

Majority of Indian females with ACS are postmenopausal, but tend to present at an early age compared to their western counterparts. Hypertension, dyslipidaemia, and age $>55$ years are factors influencing atherosclerosis as evidenced by obstructive CAD on CAG. Hypothyroidism is highly prevalent and suggests that it may play a significant role in pathogenesis of CAD.

\section{KEYWORDS}

CAD, ACS, Indian, Females, Coronary Angiography, Risk Factors.

HOW TO CITE THIS ARTICLE: Dubey TN, Kumar A. Clinical and angiographic profile of females in central India presenting with acute coronary syndrome. J.Evolution Med. Dent. Sci. 2016;5(61):4258-4262, DOI: 10.14260/jemds/2016/972

\section{INTRODUCTION}

Coronary Artery Disease (CAD) is the leading cause of morbidity and mortality in developed countries and is also becoming the leading cause of death in underdeveloped countries worldwide. For long, many believed that CAD was primarily a "man's disease". 1 CAD's impact on women traditionally has been underappreciated due to higher rates at younger ages in men. More women have died from CAD than of cancer (Including breast cancer), chronic lower respiratory disease, Alzheimer disease, and accidents combined. ${ }^{2}$ The consequences of CAD are worse in women than in men and they have higher rates of angina than do men. ${ }^{3}$ On cardiovascular computed tomography, women have been

Financial or Other, Competing Interest: None.

Submission 29-05-2016, Peer Review 09-07-2016,

Acceptance 14-07-2016, Published 30-07-2016.

Corresponding Author:

Dr. Abhinav Kumar,

40-F Block,

Gandhi Medical College

Bhopal-462001

Madhya Pradesh

E-mail: abhinavkumarshah@gmail.com

DOI: $10.14260 /$ jemds/2016/972 shown to have smaller coronary artery diameters than men do. ${ }^{4}$ Women have unique risk factors for CAD including those related to pregnancy and autoimmune disease. Women are less likely than men are to have obstructive CAD at the time of Coronary Angiography (CAG). ${ }^{5}$ Over one-half of symptomatic women without obstructive CAD continue to have signs and symptoms of ischaemia and to undergo repeat hospitalisation and coronary angiography. ${ }^{6}$ Microvascular coronary disease disproportionately affects women. Men with early CAD have higher degrees of atheroma and epicardial endothelial dysfunction whereas women with early CAD have more disease of the microvasculature. ${ }^{7}$ Currently, Indian subcontinent is experiencing rapid increase in the rates of CAD. Consequently, there is a great interest in clarifying epidemiology of coronary artery disease in this region.

There is a need to correlate the newer and conventional risk factors with precise aetiology. There has been an explosive increase in the knowledge of CAD in women in west in the past decade. There is not much published data on CAD in women in India. It was decided to undertake this study to fill in the gap with little knowledge on the profile and pattern of CAD in females residing in central India. The main aim of the study is to evaluate the presentation, prevalence of risk factors 
in females with ACS, and to correlate the impact of risk factors on presence of obstructive CAD as assessed by Coronary Angiography (CAG).

\section{MATERIALS AND METHODS}

The study was an observational study carried out at authors Department of Cardiology for 1 year from December 2014 to November 2015. The study group comprised of 92 female patients presenting with first episode of ACS (evidence of Unstable Angina (UA)/Myocardial Infarction (MI) on ECG at time of admission, and/or with raised cardiac biomarkers). The clinical presentations of patient were categorised as Unstable Angina (UA), Non-ST Elevated Myocardial Infarction (NSTEMI), and STEMI. Patients with previous evidence of coronary artery disease like previous myocardial infarction, coronary intervention, or CABG and patients taking lipid lowering therapy and those with valvular heart disease, cardiomyopathies, or myocarditis were excluded.

\section{The following Data were included for Analysis}

- Age.

- Presentation: Chest pain/ Sweating/ Breathlessness/ Nausea or Vomiting/Killip class.

- Menopausal status.

- Hypertension: Systolic blood pressure $\geq 140$ and/or diastolic $\geq 90 \mathrm{mmHg}$ and/or on anti-hypertensive treatment.

- Diabetes mellitus: Symptoms of diabetes and plasma glucose concentration $\geq 200 \mathrm{mg} / \mathrm{dL}(11.1 \mathrm{mmol} / \mathrm{L})$, or fasting blood sugar $\geq 126 \mathrm{mg} / \mathrm{dL}$ ( $7.0 \mathrm{mmol} / \mathrm{L})$.

- Addiction: Cigarette/beedi smoking/ tobacco chewing history (patients who were actively consuming tobacco/beedi or had quit within last 2 months were considered as substance abuser and patients who had quit beyond 2 months were considered in the category of no addiction).

- Dyslipidaemia was defined as the presence of any of the following:

- Total cholesterol $>240 \mathrm{mg} / \mathrm{dL}$, Triglycerides (TG) $>150$ $\mathrm{mg} / \mathrm{dL}$, Low-Density Lipoprotein (LDL) $>130 \mathrm{mg} / \mathrm{dL}$, and High-Density Lipoproteins (HDL) $<50 \mathrm{mg} / \mathrm{dL}$.

- Family history of CAD: first-degree relatives with CAD before the age of 55 years in men and 65 years in women.

- Hypothyroidism: Patients were defined as having subclinical hypothyroidism when TSH $>5.5$ micro international units/mL with normal values of T3 and T4, and as overt hypothyroidism if they were having low values of $\mathrm{T} 3$ and $\mathrm{T} 4$ or were taking tab thyroxine.

- Obesity was defined using the waist-hip ratio where a ratio $>0.85$ was considered as obese.

- $\quad$ Coronary angiography results.

Selective coronary angiogram was done using standard technique unless patient was haemodynamically unstable or with deranged renal parameters. Significant/obstructive CAD was defined as a diameter stenosis $>70 \%$ in each major epicardial artery except left main disease where a stenosis of $>50 \%$ was considered significant. Patients were classified as having Single-Vessel Disease (SVD), Double-Vessel Disease (DVD) or Triple-Vessel Disease (TVD) accordingly.

Normal vessels were defined as the complete absence $/<20 \%$ stenosis in the Left Main Coronary Artery
(LMCA), Left Anterior Descending (LAD), Right Coronary Artery (RCA), and Left Circumflex (LCx) as well as in their main branches (Diagonal, obtuse marginal, ramus intermedius, posterior descending artery, and posterolateral branch). For comparative analysis patients were categorised into two groups based on their cardiac catheterisation findings (i.e. angiographic nonobstructive cases who did not have significant stenosis in any of the coronary artery and obstructive CAD who had significant stenosis in at least one coronary artery). The risk factors included in the study were analysed and their impact on finding obstructive CAD was assessed.

The results were reported as mean \pm standard deviation for the quantitative variables and percentages for the categorical variables. The groups were compared using the chi-square test and Fisher's exact test for the categorical variables. $\mathrm{P}<0.05$ was considered as statistically significant. All the statistical analyses were carried out via Microsoft Excel and SPSS (Version 23.0).

\section{RESULTS}

The average age of females in our study was 56.7 years with majority belonging to $6^{\text {th }}$ and $7^{\text {th }}$ decade. Age profile is shown in table 1.

\begin{tabular}{|c|c|c|c|c|c|}
\hline $\begin{array}{c}\text { Age } \\
\text { (In years) }\end{array}$ & $30-39$ & $40-49$ & $50-59$ & $60-69$ & $\geq 70$ \\
\hline $\begin{array}{c}\text { Number } \\
(\% \text { age })\end{array}$ & $\begin{array}{c}2 \\
(2.2 \%)\end{array}$ & $\begin{array}{c}25 \\
(27.2 \%)\end{array}$ & $\begin{array}{c}27 \\
(29.3 \%)\end{array}$ & $\begin{array}{c}27 \\
(29.3 \%)\end{array}$ & $\begin{array}{c}11 \\
(12 \%)\end{array}$ \\
\hline \multicolumn{6}{|c|}{ Table 1: Age Profile of Females with ACS } \\
\hline
\end{tabular}

Chest pain was seen in $90.2 \%$, sweating in $82.6 \%$, breathlessness in $50 \%$, and nausea/vomiting in $31.5 \%$ of patients. $63(68.5 \%)$ belonged to Killip class I, $19(20.6 \%)$ to class II, $6(6.5 \%)$ to class III, and $4(4.4 \%)$ to class IV. Most common presentation of ACS among females was STEMI $63.1 \%$ (40.2\% had AWMI, $23.9 \%$ had IWMI), NSTEMI was observed in $5.5 \%$ of patients and UA in $30.4 \%$. Menopause is one of the most important non-modifiable risk factors for CAD in females, majority $(81.5 \%)$ of the patients in our study were postmenopausal. $62 \%$ of the females were found to be hypertensive. Hypertension was found to be significantly associated with obstructive CAD on CAG. $34.8 \%$ of the study population was found to be diabetic and prevalence of multivessel disease was higher in them. Only $2.2 \%$ of females were beedi smokers and $25 \%$ were tobacco chewers and no significant association with obstructive CAD was observed in them. $68.5 \%$ females were found to be obese based on waisthip ratio. $31.5 \%$ of female patients had a positive family history of CAD. $13 \%$ of patients had overt hypothyroidism and $23.9 \%$ of patient had subclinical hypothyroidism suggesting a high prevalence of hypothyroidism in patients with CAD in central India. $90.2 \%$ of patients were found to be having dyslipidaemia and it was the most prevalent risk factor found in the study. Low HDL was found to be the predominant form of dyslipidaemia and was seen in $78.3 \%$ of the patients followed by raised triglycerides seen in $41.3 \%$ of patients. Dyslipidaemia had statistically significant correlation with presence of significant CAD ( $p$ value=0.03). Table 2 summarises the various observations made. 


\begin{tabular}{|c|c|}
\hline Parameter & Baseline Values \\
\hline Age (mean \pm SD) & $56.7 \pm 10.43$ \\
\hline Age $\geq 55$ years (number and \%) & $47(51.08 \%)$ \\
\hline Postmenopausal (number and \%) & $75(81.5 \%)$ \\
\hline Hypertension (number and \%) & $57(62 \%)$ \\
\hline Diabetes Mellitus (number and \%) & $32(34.8 \%)$ \\
\hline Smoking/Tobacco (number and \%) & $25(27.2 \%)$ \\
\hline Obesity (W:H Ratio) (number and \%) & $63(68.5 \%)$ \\
\hline Family History (number and \%) & $29(31.5 \%)$ \\
\hline Dyslipidaemia (number and \%) & $83(90.2 \%)$ \\
\hline High Total Cholesterol (number and & $5(5.4 \%)$ \\
\hline \%) & $57(81.4 \%)$ \\
\hline Low HDL (number and \%) & $14(15.2 \%)$ \\
\hline High LDL (number and \%) & $30(42.9 \%)$ \\
\hline Triglycerides (number and \%) & $22(23.9 \%)$ \\
\hline Hypothyroidism & $12(13 \%)$ \\
\hline Subclinical (number and \%) & $59(64.2 \%)$ \\
\hline Overt (number and \%) & \\
\hline Type of ACS & \\
\hline STEMI (number and \%) & \\
\hline
\end{tabular}

\begin{tabular}{|c|c|}
\hline NSTEMI (number and \%) & $5(5.4 \%)$ \\
\hline UA (number and \%) & $28(30.4 \%)$ \\
\hline CAG Pattern & \\
\hline Normal (number and \%) & $5(5.4 \%)$ \\
\hline Insignificant (number and \%) & $21(22.9 \%)$ \\
\hline SVD (number and \%) & $29(31.5 \%)$ \\
\hline DVD (number and \%) & $16(17.3 \%)$ \\
\hline TVD (number and \%) & $21(22.9 \%)$ \\
\hline Table 2: Baseline Characteristics of Females with ACS \\
\hline
\end{tabular}

Nonobstructive lesions on CAG post ACS is more prevalent $(42.22 \%)$ in younger age group (i.e. $\leq 55$ years) as compared to patients $>55$ years of age $(14.89 \%)$ and the difference was found to be statistically significant ( $p$ value $=0.003$ ). Most common coronary artery to be involved was LAD with $58.5 \%$ of females having significant stenosis in LAD. Near similar frequency of RCA (40\%) and LCx (34.2\%) involvement was seen. LMCA disease was seen in $2.8 \%$ females. The distribution of coronary vessel involvement is summarised in table 3 .

\begin{tabular}{|c|c|c|c|c|c|}
\hline ACS & Normal n (\%) & Insignificant n (\%) & SVD n (\%) & DVD n (\%) & TVD n (\%) \\
\hline UA & $3(3.3 \%)$ & $12(13 \%)$ & $4(4.3 \%)$ & $4(4.3 \%)$ & $5(5.4 \%)$ \\
\hline NSTEMI & $2(2.2 \%)$ & $1(1.1 \%)$ & $2(2.2 \%)$ & 0 & 0 \\
\hline STEMI & 0 & $8(8.7 \%)$ & $23(25.0 \%)$ & $12(13 \%)$ & $16(17.4 \%)$ \\
\hline \multicolumn{7}{|r|}{ Table 3: CAG Pattern in Females with ACS } \\
\hline
\end{tabular}

\section{DISCUSSION}

CAD is the leading cause of death and a major cause of morbidity amongst women. Prior to the fifth decade of life, prevalence in men is greater than in women, but in the sixth decade, prevalence equalizes and in subsequent decades becomes greater in women ${ }^{8}$. The mean age of females presenting with first episode of ACS in our study population was $56.7 \pm 10.43$ years. This is somewhat comparable to other studies done in India e.g. Sharma et $\mathrm{al}^{9}(60.23 \pm 17.67$ years $)$ and Dwivedi et al ${ }^{10}(59.17 \pm 10.67$ years), but lower than in western populations as in COURAGE trial. ${ }^{11}$ ( $62 \pm 5$ years) and Hochman et al ${ }^{12}$ (69 years). Kerala ACS Registry. ${ }^{13}$ with 5,825 women amongst the 25,748 ACS patients showed that on presentation, women were approximately 5 years older than men.

Chest pain was the most common symptom at the time of presentation and was observed in $90.2 \%$ of the patients. Sweating was observed in $82.6 \%$ of the study population and complains of breathlessness in $50 \%$. Nausea/Vomiting was observed in $31.5 \%$ of the study population. Majority of the patients were in Killip's class I (68.5\%) and II (20.6\%).

Most common presentation of ACS in females was STEMI $63.1 \%$ (40.2\% had AWMI, $23.9 \%$ had IWMI), NSTEMI was observed in $5.5 \%$ of patients and unstable angina in $30.4 \%$. CREATE registry ${ }^{8}$ showed that $60 \%$ of ACS patients are constituted by STEMI while in Kerala ACS registry ${ }^{13}$ STEMI constituted only $40 \%$. In many International Registries, STEMI constitutes one-third of cases.

Menopause is one of the most important non-modifiable risk factor for CAD in females. Majority (81.5\%) of the patients in our study were postmenopausal in spite of a mean age of 56.7 years in the study population. Dwivedi et al10 in their study observed that $86.5 \%$ of the females were postmenopausal. This brings into perspective fact that menopause plays a crucial role in pathogenesis of CAD and early menopause in Indians females carries with it a significantly increased risk of early onset of cardiovascular diseases.

$62 \%$ of the study population was found to be hypertensive. Dwivedi et al 10 in their study on females in north India with CAD found that $58.5 \%$ were hypertensive. In our study, we found that $80 \%$ of the hypertensive patients and $56 \%$ of normotensive patients who underwent coronary angiography had obstructive CAD on CAG. Of those found to have multivessel CAD, $78.5 \%$ were hypertensives. When statistical tests were applied to see for correlation between hypertension and obstructive CAD, we found a statistically significant correlation between them ( $p$ value $<0.05$ ).

Diabetes is a risk factor for the presence and severity of CAD in both men and women, but carries a greater incremental risk in women completely eliminating the "female advantage". $1434.8 \%$ of the study population was found to be diabetic. It is comparable to other studies in India e.g. Dwivedi et $\mathrm{al}^{10}$ (29.5\% of females with CAD were diabetic). We found that diabetes mellitus did not have statistical significance when correlated with presence of obstructive CAD on CAG, although TVD was seen in $40 \%$ of diabetic patients and $13.3 \%$ of non-diabetic patients confirming the role of diabetes as a significant contributor to multivessel CAD.

Addiction among female patients is not as prevalent in our country as compared to the male population, although addiction is on the rise in females especially in urban population and because of its anti-estrogenic effect, it quadruples the risk of MI in young. We found that 25(27.2\%) were tobacco chewers/smoking beedi and of them only $2(2.2 \%)$ were beedi smokers, and rest $23(25 \%)$ were tobacco chewers. We did not find significant correlation between smoking/tobacco chewing and obstructive CAD ( $\mathrm{p}$ value $>0.05$ ).

Obesity was assessed using waist-hip ratio, which has been found to be a better predictor for cardiovascular disease in our study population. $68.5 \%$ females were found to be obese. Of the obese patients, $77.27 \%$ were found to have obstructive 
CAD, and $61.5 \%$ of non-obese patients were found to have obstructive CAD. Obesity was not found to have a statistically significant influence on presence of obstructive CAD as assessed by CAG.

Among females, a family history of premature CAD in a sister is associated with a 12 fold higher risk versus 6 fold for a brother and 3 fold for a parent. 15 31.5\% of female patients had a positive family history of CAD. There was no impact of a positive family history on angiographically obstructive CAD.

Overt hypothyroidism affects $\approx 3 \%$ of the adult female population. It is estimated that as many as $7 \%$ to $10 \%$ of older women have subclinical hypothyroidism. ${ }^{16}$ We found that $13 \%$ of patients had overt hypothyroidism and $23.9 \%$ of patient had subclinical hypothyroidism suggesting a high prevalence of hypothyroidism in patients with CAD in central India. Hypothyroidism was prevalent in all age groups of study population. Overt and subclinical hypothyroidism was associated with increase in total cholesterol, LDL, and triglyceride level. Patients with overt hypothyroidism were found to have significantly lower HDL cholesterol level, but the same was not observed in patients with subclinical hypothyroidism. We found that hypothyroidism though highly prevalent in female patients with CAD, it was not found to have any statistically significant association with CAD severity ( $\mathrm{p}$ value $>0.05$ ).

$90.2 \%$ of patients were found to be having dyslipidaemia and it was the most prevalent risk factor found in the study. Although, total serum cholesterol was found to be normal in majority (91.3\%) of patients, but a low HDL was found to be the predominant form of dyslipidaemia and was seen in $78.3 \%$ of the patients followed by raised triglycerides seen in $41.3 \%$ of patients. $33.3 \%$ of the patients with normal lipid profile had obstructive CAD on CAG whereas $75 \%$ of the patients with dyslipidaemia had obstructive CAD. We found that dyslipidaemia had statistically significant correlation with presence of obstructive CAD ( $p$ value $=0.03$ ).

Relative proportion of patients with nonobstructive lesions is higher in younger age group as compared to older patients $(42.22 \%$ of females $<55$ years and $14.89 \%$ of females $\geq 55$ years had insignificant lesions/normal coronaries on coronary angiography) and the difference was found to be statistically significant ( $p$ value $=0.003$ ) [Fig. 1]. Most common coronary artery to be involved was LAD. Significant LMCA stenosis was observed in $2.8 \%$ of females. Normal or insignificant disease was present in $28.3 \%$. Angiographically, the completely normal vessels post ACS has been attributed to complete recanalisation whether spontaneous/post thrombolysis.

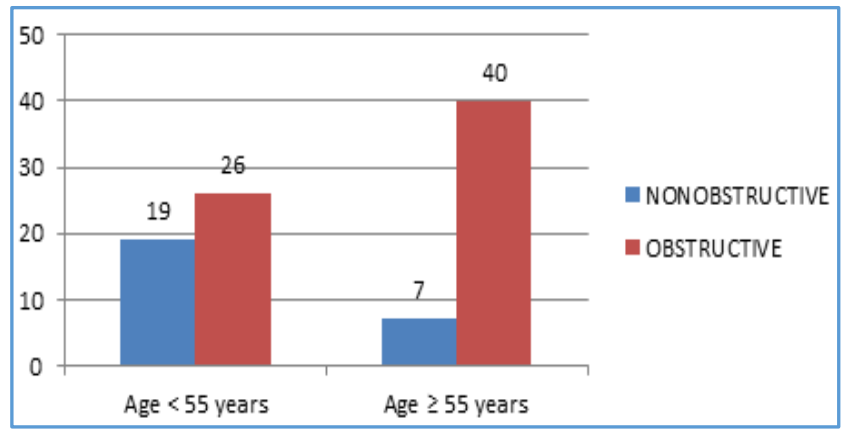

Fig. 1: Bar Chart Depicting Comparing Number of Patients with Nonobstructive and Obstructive CAG Pattern in Different Age Group
The limitation of our study was small sample size. What is needed is a large study sample with males serving as control groups to assess the differences between the presentation and profile of CAD in them.

\section{CONCLUSION}

Majority of Indian females with ACS are postmenopausal. Women especially postmenopausal with risk factors for atherosclerosis should be identified and treated aggressively to prevent devastating cardiovascular events. Hypothyroidism (Both overt and subclinical) is highly prevalent and suggests that it may play a significant role in pathogenesis of CAD. Hypertension, dyslipidaemia, and age $\geq 55$ years have significant association with obstructive CAD in Indian females. Despite high incidence of ACS at younger age, females especially of young age have lower atherosclerotic burden as understood from higher prevalence of non-obstructive CAD.

\section{REFERENCES}

1. Bedhighaus J, Leshan L, Diehr S. Coronary artery disease prevention: what's different for women? American Family of Physicians 2001;63(7):1393-401.

2. Roger VL, Go AS, Jones LDM, et al. Heart disease and stroke statistics-2012 update: a report from the American heart association. Circulation 2012;125(1): e2-e220.

3. Vaccarino V, Parsons L, Every NR, et al. Sex-based differences in early mortality after myocardial infarction. National registry of myocardial infarction 2 participants. New England Journal of Medicine 1999;341(4):217-25.

4. Dickerson JA, Nagaraja HN, Raman SV. Gender-related differences in coronary artery dimensions: a volumetric analysis. Clinical Cardiology 2010;33(2):E44-9.

5. Sharaf BL, Pepine CJ, Kerensky RA, et al. Detailed angiographic analysis of women with suspected ischaemic chest pain (pilot phase data from the NHLBIsponsored women's ischaemia syndrome evaluation study angiographic core laboratory). American Journal of Cardiology 2001;87(8):937-41.

6. Shaw LJ, Merz CN, Pepine CJ. The economic burden of angina in women with suspected ischaemic heart disease: results from the national institutes of health, national heart, lung, and blood institute sponsored women's ischaemia syndrome evaluation. Circulation 2006;114(9):894-904.

7. Han SH, Bae JH, Holmes DR, et al. Sex differences in atheroma burden and endothelial function in patients with early coronary atherosclerosis. European Heart Journal 2008;29(11):1359-69.

8. Majumder B, Haque A, Dastidar DG. Recent insights in coronary artery disease in women. Medicine Update (API India) 2011.

9. Sharma R, Bhairappa S, Prasad SR. Clinical characteristics, angiographic profile, and in hospital mortality in acute coronary syndrome patients in south Indian population. Heart India 2014;2(3):65-9.

10. Dwivedi S, Dhar M, Agarwal MP, et al. Clinical profile of coronary artery disease in women. Indian J Cardiology 2006;9:18-23. 
11. O'Gara PT. The clinical outcomes utilizing revascularization and aggressive drug evaluation trial: can we deliver on its promise? J Am Coll Cardiol 2010;55(13):1359-61.

12. Hochman JS, Tamis JE, Thompson TD, et al. Sex, clinical presentation, and outcome in patients with acute coronary syndromes. N Engl J Med 1999;341(4):226-32.

13. Patel A, Mohanan PP, Huffmman MD. Gender differences in the presentation, diagnosis, and management of acute coronary syndrome in Kerala, India: result from the Kerela ACS registry. Glob Heart 2015;10(4):273-80.
14. Jousilahti P, Vartiainen E, Tiromilehto J, et al. Sex, age, cardiovascular risk factors, and coronary heart disease: a prospective follow up study of 14,786 middle-aged men and women in Finland. Circulation 1999;99(9):1165-72.

15. Beverly RK, Siskovick D, Schwartz S, et al. Family history and risk of myocardial infarction in young women. Circulation 1996;93:635.

16. Canaris GJ, Manowitz NR, Mayor G, et al. The Colorado thyroid disease prevalence study. Arch Intern Med 2000;160(4):526-34. 